\title{
Der weiße Kittel ist in der Psychiatrie nicht mehr zeitgemäß - Pro
}

\author{
The Psychiatrist's White Coat is Outdated - Pro
}

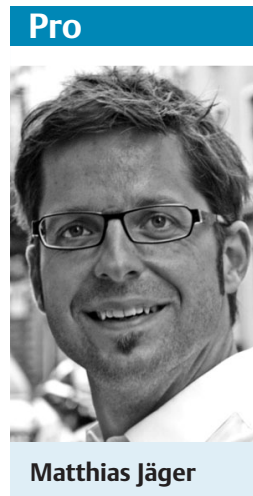

Mit Ausnahme hygienischer Aspekte in einer somatischen Untersuchungs- oder Behandlungssituation erfüllt der weiße Kittel in der Psychiatrie keinerlei praktische Funktion. Hingegen fungiert er als Symbol für den Status und die Kompe-

tenz der Ärztin oder des Arztes* und hat dadurch eine direkte Auswirkung auf die therapeutische Beziehung. Neben möglichen positiven Aspekten wie Kenntlichkeit, Vertrauen und Sicherheit vermittelt der weiße Kittel insbesondere die Gewissheit, dass ein grundlegender Unterschied zwischen Arzt und Patient besteht, die der weiteren Interaktion einen asymmetrischen Einschlag verleiht. Dies ist im Prinzip in allen medizinischen Fachdisziplinen, jedoch insbesondere in der Psychiatrie (wie auch der Pädiatrie) relevant, da die therapeutische Beziehung hier nicht nur das Vehikel zur Vermittlung von therapeutischen Interventionen, sondern für den Behandlungserfolg auch ein hochrelevanter Wirkfaktor per se ist [1]. Nun geht es nicht darum, faktische Differenzen zwischen den Rollen von Arzt und Patient zu verschleiern oder das Selbstverständnis des Arztes als Fachperson mit umfassender Kompetenz in Gesundheitsfragen zu entwerten. Vielmehr liegt der Fokus des Interesses im Zusammenhang mit dem äußerlichen Erscheinungsbild und dem Auftreten des Arztes auf den Auswirkungen auf ein therapeutisch zentrales Phänomen, eben der therapeutischen Beziehung. Derzeit wird das Konzept des „Shared-decision-making“ in der Medizin als Goldstandard betrachtet [2]. Dieses thera-

\footnotetext{
* Im Folgenden wird zur besseren Lesbarkeit für alle Personenbezeichnungen die männliche Form gewählt, die sowohl männliche als auch weibliche Personen einschließt.
}

peutische Beziehungsmodell basiert auf einem dialektischen, gleichberechtigten Austausch zwischen Arzt und Patient, der idealerweise in eine gemeinsame Entscheidungsfindung in Bezug auf das weitere Vorgehen mündet. Der Arzt bringt inhaltlich sein Fachwissen und seine Erfahrung ein, der Patient beteiligt sich mit seinen Vorkenntnissen, Präferenzen, Werten und früheren Erfahrungen. Ein formaler Rahmen, der a priori traditionelle Machtverhältnisse verdeutlicht (pointiert: der Patient im Flügelhemd, der Arzt im weißen Kittel), kann diese „Begegnung auf Augenhöhe“ erheblich erschweren.

Um nun gerade diesen Ansatz („der Arzt weiß schon, was für den Patienten am besten ist") hier nicht zu replizieren, empfiehlt sich ein Blick in die Literatur zur Perspektive von Patienten auf Erscheinungsbild und Auftreten des Arztes. Bei Ärzten im Allgemeinen wurde noch in den 1980er- und 90er-Jahren ein weißer Kittel über formeller Kleidung bevorzugt [3, 4], während neuere Untersuchungen zu unterschiedlichen Resultaten kommen. Studien aus England und Neuseeland ergaben eine Präferenz für semiformelle Kleidung und einen weniger hohen Stellenwert des weißen Kittels [5, 6] gegenüber einer amerikanischen Untersuchung, in der drei Viertel der Probanden für formelle Kleidung mit weißen Kitteln plädierten [7].

Psychiater in formeller Kleidung werden von ihren Patienten als kompetenter, jedoch weniger freundlich, verständnisvoll und zugänglich erlebt, während legere Kleidung eine umgekehrte Wirkung hat $[8,9]$. Psychiatrische Patienten in stationärer Behandlung bevorzugen elegant gekleidete Ärzte ohne weißen Kittel. Verschiedene Autoren empfahlen daher ein elegantes, nicht zu formelles Auftreten, um ein möglichst symmetrisches Beziehungsmuster zu ermöglichen, Autonomie und Selbstbestimmung zu fördern und regressives Verhalten zu reduzieren [1012]. Die Teilnehmer einer amerikanischen Studie bevorzugten legere Kleidung bei ihren Ärzten und waren zu 96\% der Meinung, dass Psychiater keine weißen Kittel tragen sollten, obwohl 58\% angaben, dass dies keinen Unterschied für die therapeutische Beziehung bedeuten würde [13]. Insgesamt scheint das Erscheinungsbild für Ärzte ein wichtigeres Thema zu sein, als dies bei Patienten der Fall ist $[4,12$, 13].

Die Beurteilung des Erscheinungsbildes und Auftretens von Psychiatern scheint also in Zusammenhang zu stehen mit regionalen und institutionskulturellen Gepflogenheiten sowie mit dem Behandlungssetting. Ein konsistentes Ergebnis mehrerer Untersuchungen besagt, dass formellere Kleidung bzw. der weiße Kittel Kompetenz vermittelt, jedoch Distanz aufbaut und die Festigung einer tragfähigen therapeutischen Beziehung erschweren kann. Psychiatrische Patienten bevorzugten eher einen semiformellen eleganten Kleidungsstil ohne weißen Kittel. Im stationären Setting scheinen weiße Kittel noch eher akzeptiert zu sein als in der ambulanten Versorgung. In sämtlichen bisherigen Studien wurden zu diesem Thema jedoch Patienten befragt, die sich zum Befragungszeitpunkt bereits in psychiatrischer (meist stationärer) Behandlung befanden. Daher kann ein Bias angenommen werden, in dem Sinne, dass sich die Probanden an ihrem bekannten Bild von einem Psychiater orientieren konnten und daher eher für den Status quo argumentierten. Um diesen möglichen Einfluss auszuschließen, müssten andere Gruppen als diejenigen in gegenwärtig stationärer Behandlung befragt werden (z.B. eine repräsentative Stichprobe aus der Allgemeinbevölkerung, psychiatrische Patienten bei niedergelassenen Psychiatern ohne frühere Erfahrung mit stationärer bzw. institutioneller Behandlung, somatische Patienten ohne Psychiatrieerfahrung).

Die Rolle des Psychiaters hat sich seit Beginn der Etablierung der Psychiatrie, insbesondere jedoch in den letzten 50 Jahren, grundlegend geändert. Die Integra- 
tion der Psychotherapie in die Facharztausbildung sowie die seit den Psychiatriereformen dem ärztlich-psychiatrischen Aufgabenfeld inhärenten sozialpsychiatrischen Behandlungs- und Versorgungsaspekte haben die Tätigkeitsbereiche von Psychiatern erheblich erweitert. Wo in den meisten Bereichen der somatischen Medizin eine Fokussierung auf eine eher versachlichte Krankheitsentität im Rahmen einer distanzierten Geschäftsbeziehung grundsätzlich möglich ist (die Frage der Wünschbarkeit bleibt hier ausgeklammert), kommt die Psychiatrie schwerlich an der zentralen Bedeutung der therapeutischen Beziehung für eine erfolgreiche Behandlung vorbei. Das professionelle Auftreten und Verhalten von Psychiatern, aber auch anderen in der Psychiatrie tätigen Fachpersonen, sollte daher Gegenstand stetiger Aufmerksamkeit und Reflexion sein. Dies stets mit dem Fokus auf der Ermöglichung und Förderung einer vertrauensvollen, tragfähigen und menschlich ausbalancierten therapeutischen Beziehung. Ob das symbolisch aufgeladene Element des weißen Kittels hierbei hilfreich ist, bleibt angesichts oben ausgeführter Ergebnisse zur subjektiven Perspektive psychiatrischer Patienten fraglich.

\section{Literatur}

1 Priebe S, McCabe R. Therapeutic relationships in psychiatry: the basis of therapy or therapy in itself? Int Rev Psychiatry 2008; 20: $521-526$

2 Joosten EA, DeFuentes-Merillas L, de Weert $G H$ et al. Systematic review of the effects of shared decision-making on patient satisfaction, treatment adherence and health status. Psychother Psychosom 2008; 77: 219 226

3 Dunn JJ, Lee TH, Percelay JM et al. Patient and house officer attitudes on physician attire and etiquette. Jama 1987; 257: 65-68

4 Gjerdingen DK, Simpson DE, Titus SL. Patients' and physicians' attitudes regarding the physician's professional appearance. Arch Intern Med 1987; 147: 1209-1212

5 Lill MM, Wilkinson TJ. Judging a book by its cover: descriptive survey of patients' preferences for doctors' appearance and mode of address. BMJ 2005; 331: $1524-1527$

6 Palazzo S, Hocken DB. Patients' perspectives on how doctors dress. J Hosp Infect 2010; 74: $30-34$

7 Rehman SU, Nietert PJ, Cope DW et al. What to wear today? Effect of doctor's attire on the trust and confidence of patients. Am J Med 2005; 118: 1279-1286

8 Nome Eikhom M, Torsaeter M, Wik G. Psychiatric patients: views on psychiatrists' dress and address. Nord J Psychiatry 2006; 60: $270-274$

9 Tham SW, Ford TJ. Staff dress on acute psychiatric wards. Journal of Mental Health 1995; 4: 297 - 299
10 Gledhill JA, Warner JP, King M. Psychiatrists and their patients: views on forms of dress and address. Br J Psychiatry 1997; 171: 228-232

11 McGuire-Snieckus R, McCabe R, Priebe S. Patient, client or service user? A survey of patient preferences of dress and address of six mental health professions. Psychiatric Bulletin 2003; 27: 305-308

12 Rajagopalan $M$, Santilli $M$, Powell $D$ et al Mental health professionals' attire. Aust N Z J Psychiatry 1998; 32: 880-883

13 Nihalani ND, Kunwar A, Staller J et al. How should psychiatrists dress? - A survey. Community Ment Health J 2006; 42: 291 - 302

Sie haben eine eigene Meinung zu diesem Thema? Dann schreiben Sie uns an: psychiat-praxis@thieme.de!

\section{Korrespondenzadresse}

\section{Dr. med. Matthias Jäger}

Psychiatrische Universitätsklinik Zürich Lenggstraße 31

8032 Zürich, Schweiz

matthias.jaeger@puk.zh.ch

\section{Bibliografie}

DOI http://dx.doi.org/

10.1055/s-0034-1387556

Psychiat Prax 2015; 42: 122-123

(c) Georg Thieme Verlag KG

Stuttgart - New York

ISSN 0303-4259 